\title{
Cultura institucional y perspectiva de género en las corporaciones de seguridad pública en México*
}

\author{
[Artículos]
}

\author{
Cristina Rumbo Bonfil ${ }^{* *}$ \\ Ángela Paola Contreras Álvarez ${ }^{* * *}$
}

Recibido: 10 febrero de 2021

Aceptado: 6 de mayo de 2021

Citar como:

Rumbo Bonfil, C. y Contreras Álvarez, A. P. (2021). Cultura institucional y perspectiva de género en las corporaciones de seguridad pública en México. Revista IUSTA, (55). https://doi.org/10.15332/25005286.6854

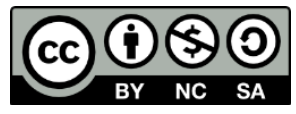

\footnotetext{
* Este artículo es resultado de la investigación "Verdad jurídica y verdad histórica. Reconstrucción de violencia contra las mujeres en Córdoba" (código: 48562), desarrollada en colaboración con el grupo de investigación Red Internacional de Política Criminal Sistémica y la Fundación Universitaria del Área Andina, Colombia. ** Doctora en Derecho y Globalización por la Universidad Autónoma del Estado de Morelos, México; coinvestigadora del grupo de investigación Red Internacional de Política Criminal Sistémica Extrema Ratio UN de la Universidad Nacional de Colombia y socia fundadora de la Academia Latinoamericana de Derecho Penal y Penitenciario. Actualmente es directora asociada de la Escuela de Ciencias Sociales y Gobierno del Tecnológico de Monterrey, Campus Chihuahua. Correo electrónico: lic.cristinarb@gmail.com, cristina.rumb@tec.mx; ORCID: https://orcid.org/0000-00026724-5147

*** Abogada de la Universidad Santo Tomás, Seccional Tunja, Colombia, licenciada en Ciencias Sociales de la Universidad Pedagógica y Tecnológica de Colombia (UPTC), especialista en Derecho Constitucional de la Universidad Nacional de Colombia y magíster en Derechos Humanos de la UPTC. Actualmente es docente de medio tiempo del Departamento de Humanidades de la Fundación Universitaria del Área Andina. Correo electrónico: acontreras34@areandina.edu.co; ORCID: https://orcid.org/00000002-3933-090X
} 


\section{Resumen}

La brecha de género no solo está presente en aquellas instituciones que históricamente han tenido una tradición laboral masculina. Aunque la presencia y participación de las mujeres en espacios públicos actualmente es más visible, las posiciones de poder, mando o jerarquía siguen siendo asignadas con prelación al género masculino, que ocupa puestos de jefatura en mayor medida que el femenino.

Resulta fundamental posicionar dentro de la agenda estatal y social la construcción de una cultura institucional basada en la perspectiva de género y en la transversalidad de las condiciones laborales y de acceso igualitarias en la administración pública estatal, para identificar áreas de oportunidad donde es preciso realizar un balance y un diagnóstico sobre las conductas o prácticas que incentivan la desigualdad, como sucede en las corporaciones policiales.

Palabras clave: perspectiva de género, policía, seguridad pública, violencia.

\section{Institutional culture and gender perspective in Mexico's public security forces}

\section{Abstract}

The gender gap is not only observed in those institutions that historically have had a male labor tradition. Although the presence and participation of women in public spaces is currently more visible, positions of power, command or hierarchy continue to be assigned more favorably to the male gender, who hold more leadership positions than the female gender.

It is essential to position within the state and social agenda, the construction of an institutional culture based on the gender perspective and the mainstreaming of labor conditions and equal access in the state public administration, in order to identify areas of opportunity where it 
is required to make a balance and a diagnosis on the behaviors or practices that encourage inequality, as is the case in police forces.

Keywords: gender perspective, police, public security, violence.

\title{
Cultura institucional e perspectiva de gênero nas corporações de segurança pública no México
}

\section{Resumo}

\begin{abstract}
A lacuna de gênero não somente está presente nas instituições que historicamente têm uma tradição de trabalho masculino. Embora a presença e a participação das mulheres em espaços públicos atualmente são mais visíveis, as posições de poder, liderança ou hierarquia continuam sendo designadas de preferência ao gênero masculino, que ocupa postos de chefia em maior medida que o feminino. É fundamental posicionar dentro da agenda estatal e social a construção de uma cultura institucional baseada na perspectiva de gênero e na transversalidade das condições trabalhistas e de acesso igualitárias na administração pública estatal para identificar áreas de oportunidade em que é preciso realizar um balanço e um diagnóstico sobre os comportamentos e as práticas que incentivam a desigualdade, como ocorre nas corporações policiais.
\end{abstract}

Palavras-chave: perspectiva de gênero, polícia, segurança pública, violência.

\section{Introducción}

En las últimas dos décadas, la expresión "violencia de género" ha tomado mayor relevancia en las agendas públicas y privadas del país y del mundo entero. Adentrarse en la importancia de la perspectiva de género frente a la violencia de género se ha convertido en una política de carácter obligatorio al interior de las instituciones públicas a nivel nacional y regional. La relevancia de este concepto no consiste únicamente en ser una estructura de pensamiento que "está de moda" o una imposición para las 
instituciones, sino que obedece a una imperante necesidad de adoptar medidas preventivas y de atención ante un problema real que afecta a miles de mujeres en México.

La visibilización de la violencia de género ha permitido identificar que subsiste en los contextos más cercanos: la familia, la escuela y la sociedad. Comúnmente somos parte de una cultura que ha objetivizado el papel de la mujer en los diversos roles sociales y que ha normalizado las diferencias de trato y desarrollo entre hombres y mujeres. Por ello, recientemente ya no solo se habla de violencia, sino, de forma amplia, de "violencias", con el propósito de visibilizar todos aquellos espacios de poder donde las mujeres suelen ser víctimas de distintos tipos de abuso, normalizados e institucionalizados durante años. Por violencia contra la mujer se puede entender:

[...] cualquier acción u omisión, que le cause muerte, daño o sufrimiento físico, sexual, psicológico, económico o patrimonial por su condición de mujer, así como las amenazas de tales actos, la coacción o la privación arbitraria de la libertad, bien sea que se presente en el ámbito público o en el privado. (Alarcón, Díaz y Hernández, 2019, p. 86)

De acuerdo con datos del Secretariado Ejecutivo del Sistema Nacional de Seguridad Pública (SESNSP, 2020a) y del Instituto Nacional de Estadística y Geografía (Inegi, 2017), en México diez mujeres son asesinadas diariamente. De acuerdo con la Red por los Derechos de la Infancia en México (Redim), uno de cada diez feminicidios se comete contra niñas y adolescentes menores de 17 años. Mientras que la Encuesta Nacional sobre la Dinámica de las Relaciones en los Hogares (ENDIREH, 2017) muestra que, de las mujeres mayores de 15 años, $66 \%$ han sufrido algún incidente de agresión física (34 \%), emocional (49 \%), económica (29\%) o sexual 
(41.3\%), y que dichas agresiones vienen tanto de agresores conocidos como desconocidos, tanto en el espacio privado como en el público.

En América Latina la violencia de género ha aumentado peligrosamente, de acuerdo con la Cepal (2018), "solo en 2018 al menos 3529 mujeres fueron víctimas de feminicidio en 25 países de América Latina y el Caribe”. La región es la segunda más peligrosa en el mundo para las mujeres, solo superada por África. Desde el sur en Argentina, donde se detectaron 3013 casos entre 2010 y 2019, hasta el norte en México, donde en 2019 se registraron 1006 casos de feminicidio - cifra que situó al país azteca como el de más casos en la zona junto a Brasil (Connectas, 2020) - . Estas cifras determinan la "normalización" de los preceptos de todos los tipos de violencia a la que se enfrentan las mujeres en la mayoría de los espacios públicos y privados en los que se desarrollan.

Sin embargo, a pesar de que parezca común y cotidiano, el término "violencia de género" no fue acuñado hace mucho tiempo por la comunidad internacional. La primera ocasión que se hizo referencia al concepto de violencia de género fue a partir de 1993, a través de la Conferencia Mundial de los Derechos Humanos de Viena (ONU, 1993), que declara en el punto 18:

Los derechos humanos de la mujer y de la niña son parte inalienable, integrante e indivisible de los derechos humanos universales. La plena participación, en condiciones de igualdad, de la mujer en la vida política, civil, económica, social y cultural en los planos nacional, regional e internacional y la erradicación de todas las formas de discriminación basadas en el sexo son objetivos prioritarios de la comunidad internacional. La violencia y todas las formas de acoso y explotación sexuales, en particular las derivadas de prejuicios culturales y de la trata internacional de personas, son incompatibles con la dignidad y la valía de la persona humana y deben ser eliminadas. Esto puede lograrse con 
medidas legislativas y con actividades nacionales y cooperación internacional en esferas tales como el desarrollo económico y social, la educación, la atención a la maternidad y a la salud y el apoyo social. (ONU, 1993, pp. 4-5)

La visibilización de la violencia de género en todas sus expresiones ha sido trascendental para transitar hacia procesos de igualdad entre mujeres y hombres, pues es imposible hablar de igualdad sin reconocer que durante siglos no han existido condiciones igualitarias entre los géneros, las luchas que han enfrentado las mujeres frente al reconocimiento pleno de sus derechos políticos, civiles, sexuales, reproductivos o laborales -solo por citar algunos-, han dado muestra inequívoca de esa inequitativa condición, que las ha situado ante la imposibilidad de hacer pleno uso de sus derechos internacionalmente reconocidos (Feria, 2020).

Actualmente, a través de la lucha contra la violencia de género, diversos organismos internacionales reconocen la necesidad de que las mujeres cuenten con pleno acceso a una vida libre de violencia, una obligación compartida por los Estados y sus instituciones, que deben no solo garantizarlo u otorgarlo, sino generar condiciones, brindar herramientas eficaces y realizar acciones afirmativas, que propicien acciones igualitarias para mujeres y hombres, con cero tolerancia a cualquier forma de discriminación, violencia o condición inequitativa dentro y fuera de estas (Maldonado, 2017).

Los organismos internacionales son precisamente quienes se han encargado no solo de visibilizar y reconocer el acceso libre a una vida sin violencia, sino también al reconocimiento de otras condiciones de agresión y abuso, específicamente sobre las medidas que deben incorporar los Estados. Al respecto, Calle (2013) menciona: 
la mujer recientemente ha sido reconocida, en instancias nacionales e internacionales, como un sujeto de especial protección, debido a las vulneraciones que estas han tenido que soportar a través del tiempo por razones de estereotipos de género negativos y perjudiciales. A raíz del reconocimiento de esta problemática, se han creado instrumentos y organizaciones para su protección. A nivel internacional han surgido varios elementos, pero, puntualmente, la CEDAW y la Convención Belém do Pará; por su parte, Colombia en la Ley 1257 de 2008 define lo que se entiende por violencia contra la mujer y además reforma disposiciones que se encuentran en el Código Penal, el Código de Procedimiento Penal y la Ley 294 de 1996, para que se encuentren en armonía con la nueva definición y protección que allí se aporta. (Citado en Gómez y Herrera, 2018, p. 89)

Sin embargo, a pesar de que existe un pleno reconocimiento tanto nacional como internacional de estos derechos, todavía queda un largo recorrido para garantizar el pleno ejercicio de estos (Blanco, 2020), de manera que sea posible establecer condiciones igualitarias en los diversos espacios donde se desarrollan: personal, social, familiar o laboral. Respecto a este último ámbito es preciso mencionar que la cultura institucional con perspectiva de género cumple una función fundamental.

El hecho de que dentro de las instituciones públicas existan condiciones de "piso parejo" propicia que, además de que se cumplan los acuerdos convenidos respecto a los derechos humanos universalmente reconocidos, se generen espacios seguros, competitivos y con miras a generar una cultura institucional que permita contar con un sistema de respeto y atención a los derechos de las mujeres y al ejercicio de las diversas funciones que se desarrollan a través de una perspectiva de género, compartida por todos los miembros de las instituciones públicas estatales. 
En las últimas décadas del siglo XX, se aceleró la interpelación de un sistema de relaciones sociales de género basado en cierta organización que atravesaba tanto la esfera de lo público como la de lo privado. Hasta entonces, parecía natural que los hombres participaran más activamente que las mujeres en el mundo público, incluyendo los asuntos del Estado, de la economía y la producción de artes y ciencias. A las mujeres, por su parte, se les asignaba la responsabilidad del funcionamiento eficaz del ámbito privado, en el cotidiano y silencioso mantenimiento del hogar y de sus miembros (Faur, 2004, pp. 23-24).

Esta condición de "irrupción” precisamente ha dificultado el acceso de las mujeres a los espacios públicos, pues ha generado que los hombres se sientan "invadidos" en actividades que antes eran destinadas única y exclusivamente para ellos, tanto en lo público como en lo privado. Es a través de la reivindicación de estos espacios que cobra importancia la equidad de género, como mecanismo para generar condiciones de piso parejo.

$\mathrm{Al}$ respecto, Eleonor Faur (2004) menciona:

la proliferación del uso de la categoría de género contribuyó no solo a acelerar un cambio de paradigma en los estudios sobre la situación de las mujeres, sino también a expandir herramientas para su aplicación técnico-política. Entre los aportes más significativos de esta nueva conceptualización, se destaca la incorporación de los hombres en el foco del problema, en especial, a través del análisis sobre el tipo de relación que se establece y reproduce entre los géneros en distintas esferas de la vida social. (p. 25)

En este sentido, la condición de género ha posibilitado, en algunos de los casos exitosos, que existan más espacios y lugares de ocupación para el género femenino. Al mismo tiempo, esto ha ido generando políticas 
públicas que incentivan (u obligan) esa incorporación, desde las famosas cuotas de género, hasta la transversalización robusta de consideraciones de género a través de todos los componentes. Para crear un cambio, el nuevo marco normativo de la sociedad tendrá que abordar los impedimentos estructurales para la igualdad de género y el logro de los derechos de las mujeres (ONU, 2013).

De acuerdo con la Comisión Nacional de Derechos Humanos (CNDH, 2017), la participación de las mujeres mexicanas en el mercado de trabajo se ha incrementado lentamente, pero aún se encuentra por debajo del promedio de la Organización para la Cooperación y el Desarrollo Económicos (OCDE), que es de $67 \%$, y “de otros países que son referente de mayores avances en este tema, como Chile, donde la tasa es de $66 \%$, Colombia con 63 \% y Estados Unidos, en donde la participación económica de la mujer es de $68 \%$ " (CNDH, 2017). La incorporación de las mujeres en la participación de las diferentes esferas donde se desarrollan social, política, educativa, laboral, familiar, entre otras, no ha sido fácil y mucho menos pacífica.

Alrededor del mundo, muchas mujeres en distintos escenarios han tenido que sufrir violencia estructural, debido a su condición de género o como parte del desarrollo personal y profesional que desempeñan; así como una fuerte resistencia por parte de los varones al reconocimiento de sus derechos y a la visibilización de todos los tipos de violencia de los cuales son víctimas. Esto se debe a una estructura en la que una mujer puede ser víctima de un conjunto de violencias sistematizadas y en muchos casos normalizadas por la misma sociedad como consecuencia del régimen patriarcal.

Los constantes ataques que la comunidad feminista ha recibido en los últimos años dan cuenta del temor generalizado al cambio, en consonancia 
con la restitución de los derechos que por siglos han sido negados a las mujeres. En este sentido, es imprescindible hablar de la violencia contra las mujeres, que ha obligado precisamente a incentivar el discurso sobre género. Estas violencias se desarrollan bajo el amparo de la institucionalización de nuestras autoridades, que revictimizan a las mujeres en México y en América Latina, en general.

Este foco en la violencia de género además muestra que las agresiones físicas, verbales, emocionales y psicológicas han aumentado de manera alarmante en los últimos años, bajo la premisa de ser una violencia arraigada en la condición de género que obedece a un alza en las conductas de tipo doloso o al acelerado incremento del crimen organizado trasnacional de América Latina, que al menos en México ha elevado los niveles de incidencia delictiva. En los últimos dos años (2018 y 2019) se superaron de forma gradual las cifras de delitos, lo que llevó a considerarlos como los más violentos de la historia moderna del país (Díaz, 2019).

En palabras de Huertas (2012):

los actos de agresión pueden provenir de entes estatales o de particulares que conforman las diferentes esferas de la vida de las mujeres, y deben contemplarse como un fenómeno relevante por las diferentes y graves secuelas que puede generar para las personas que directamente e indirectamente puede afectar. (p. 103)

De manera que es necesario que el Estado siga ejerciendo como agente de cambio, pues mientras las políticas públicas en torno a la visibilización de la violencia de género no sean adoptadas por las autoridades y sus entes jurídicos, difícilmente existirá una implementación social en los diversos grupos focales, desde donde surge y se normaliza la violencia. 


\section{Violencia institucionalizada: la policía como agente de violencia}

Como hemos expuesto, los distintos tipos de violencia de género existentes se encuentran arraigados en una conducta que se ha perpetuado, en muchos casos, gracias a las propias instituciones; además, los hombres han entablado un proceso de resistencia al sentir un proceso de irrupción en los espacios que tradicionalmente eran destinados para el sexo masculino, que ha aumentado esas violencias en contra de las mujeres. A partir de este contexto, en las secciones subsiguientes del artículo nos ocuparemos de las corporaciones de seguridad pública, donde las circunstancias mencionadas se han desarrollado tradicionalmente, lo que ha hecho aún más grande la brecha de género, al menos desde el ámbito laboral.

Es importante mencionar que la brecha de género no se observa únicamente en aquellas instituciones donde históricamente se ha dado una tradición laboral masculina, sino que es una condición que subsiste en la administración pública en general. Pues, aunque la presencia de mujeres en espacios públicos puede ser visible, es imposible negar que las posiciones de poder, mando o jerarquía siguen siendo ocupadas en mayor medida por el género masculino.

Por consiguiente, resulta fundamental posicionar dentro de la agenda estatal y social la construcción de una cultura institucional basada en la perspectiva de género y en la transversalidad de las condiciones laborales y de acceso igualitarias en la administración pública estatal, para identificar áreas de oportunidad donde es requerido realizar un balance y un diagnóstico sobre las conductas o prácticas que incentivan que no haya un piso parejo, como sucede en las corporaciones policiales. 
Dentro de la administración pública estatal las mujeres son quienes tienen que sortear toda clase de dificultades que hacen más complicada la carrera de ascenso dentro de las estructuras de jerarquización laboral; pues además de la carga laboral que exige el puesto que se desea desempeñar, se debe contemplar que en muchos espacios laborales las mujeres sufren de acoso tanto sexual como laboral, y para el caso específico de las trabajadoras mexicanas, las profesionales no solo ganan dos veces menos que sus colegas masculinos, sino que también tienen niveles de agotamiento emocional superiores, lo que las hace más vulnerables a sufrir el síndrome de burnout (Moreno et ál., 2006).

El estudio "Autoestima, agotamiento y compromiso laborales en directivos mexicanos", de la escuela de medicina de la Universidad Panamericana (idc Online, 2014), señala que en promedio las mujeres dedican 3.5 horas diarias a la familia, mientras que los hombres solo disponen 2.8 horas para esta labor. Asimismo, el Instituto Nacional de Estadística y Geografía afirma que en promedio ellas dedican entre 10 y 20 horas semanales más que los hombres a actividades domésticas, y entre 8 y 15 horas más al cuidado de otros. Frente a este panorama resulta urgente garantizar que el número de espacios laborales tenga paridad, independientemente de si se considera un trabajo diseñado para hombres, como es el caso del ámbito policial.

Mientras que de los hombres se esperan cualidades asociadas a la valentía, fuerza, agresividad o dinamismo, a las mujeres usualmente se les vincula a condiciones de inestabilidad emocional, sumisión, miedo o debilidad. Uno de los principales factores que se piensan como obstáculos para la incorporación de mujeres policías es precisamente el nivel de fuerza o destreza que se asocia con una "debilidad" del sexo femenino, y que, por supuesto, es un aspecto negativo que justifica el hecho de que no se 
contrate el mismo número de mujeres que de hombres, o que se utiliza para favorecer a los hombres en la asignación de puestos de mando o jerarquía en la toma de decisiones.

Este tipo de características centradas en estereotipos de género asocian las "cualidades femeninas" con una debilidad de las mujeres en las actuaciones policiales, lo que deja en un nivel secundario los aspectos positivos que pueden generar su incorporación, y limitan la función policial y de prevención de la delincuencia a un aspecto netamente de fuerza. Además, se pasa por alto que las mujeres policías pueden llegar a tener el mismo índice de fuerza que sus colegas masculinos y que tener más éxito al distender el comportamiento violento o agresivo en general.

De acuerdo con datos de la Encuesta Nacional de Estándares y Capacitación Profesional Policial (Inegi, 2018), en México existían hasta el 2018 un total de 384 mil elementos de policía (incluyendo policías de los tres niveles: federal, estatal y municipal, así como ministerial federal y estatal), mientras que el portal digital del periódico Animal Político (Ángel, 2019) refiere que, mediante una consulta de transparencia, se identificó hasta 2019 un total de 330776 policías federales y locales activos. El dato más relevante del estado de la fuerza policial mexicana es que del número total de elementos apenas el $20 \%$ son mujeres policías. Esto significa que hasta la fecha el reclutamiento policial se sigue concentrando en el número de hombres que se logra captar.

Si bien es cierto que los procesos de reclutamiento están dirigidos de forma abierta tanto a hombres como mujeres, los porcentajes de convocatoria se centran en captar un mayor número de varones para incorporarse a la institución de seguridad pública. De acuerdo con el estudio "Ser mujer policía”, realizado por la organización de la Sociedad Civil Causa en Común (Déziga, 2019), el porcentaje de mujeres policías 
que aprueban los controles de confianza es mayor, en comparación con la proporción de hombres.

Por consiguiente, las instituciones de seguridad pública podrían incentivar con mayor interés el reclutamiento e incorporación de mujeres, considerando que el porcentaje de éxito en los resultados de las pruebas de admisión es mayor. Además, los procesos de reclutamiento y la aplicación de exámenes de control de confianza tienen un costo muy alto para la corporación, con resultados con un margen amplio de resultados negativos (no aprueban) frente a los resultados de los evaluados con resultados positivos (sí aprueban). En los mejores casos, el rango de éxito de aprobación va de 6 o 7 aprobados de cada 10 personas evaluadas (SESNSP, 2020b).

Pero las evaluaciones de control de confianza no son el único requisito indispensable para la incorporación a la fuerza pública, una vez realizados los filtros de selección, el o la aspirante tienen que iniciar un curso de Formación Inicial para Policía Preventivo, con una duración de 972 horas/clase distribuidas en 30 materias tanto teóricas como prácticas. Una parte de ese contenido se encuentra orientado en la enseñanza de técnicas y tácticas policiales (puestas a disposición, detenciones, traslados, uso de la fuerza), que erróneamente se conciben como manejo y adiestramiento exclusivos de la fuerza física, habilidades donde se espera que los varones estén mejor cualificados.

Sin embargo, se ha demostrado que las mujeres policías tienen menos probabilidad de usar la fuerza, o de ser acusadas por uso excesivo de esta. Las mujeres policías presentan menos probabilidad de ser acusadas o denunciadas por ciudadanos, su presencia incluso reduce el uso de la fuerza en otros compañeros policías hombres. De manera que resulta esencial contar con mujeres policías dentro de cualquier corporación 
policial, pues no solo se trata de una condición de género y de incentivar los mecanismos de inclusión, igualdad laboral y transversalidad, sino también de promover una serie de beneficios que obtiene la institución a su incorporación.

\section{La formación policial como primera barrera de incorporación de mujeres}

Solo hasta mediados del siglo XIX las mujeres fueron integradas -aunque de manera informal- a las labores policiales en Estados Unidos, específicamente en 1845 , donde eran conocidas como matronas. "Pese a todo, las mujeres siempre fueron consideradas como trabajadores de segunda y fueron cargadas de trabajos menores por parte de las corporaciones policiales. Será por ejemplo hasta 1968 cuando se les permitirá conducir una patrulla” (Arteaga, 2000, p. 75).

En México las mujeres fueron admitidas en la policía en el año 1930, en lo que se llamó Policía Femenina y de Intérpretes, que posteriormente tuvo otras denominaciones (Arteaga, 2000, p. 76). Al igual que en Estados Unidos, en México la incorporación de las mujeres dentro de los cuerpos de seguridad pública no se dio bajo las mismas condiciones ni atribuciones que los hombres, los primeros grupos o escuadrones policiales femeninos fueron considerados la "cara bonita o elegante" de la policía.

La incorporación de la primera mujer en una corporación de seguridad pública del Estado mexicano, en ese ya lejano 1969, fue el origen del Cuerpo Femenino de Vigilancia y Protección de la Ciudad de México (Villasana, 2017), un grupo de mujeres que inicialmente tuvieron funciones de guía de turistas y de cuidado de parques y plazas públicas. Actualmente, las mujeres han ido paulatinamente ganando más espacios dentro de las corporaciones policiales, no solo con una incorporación 
plena que ha posibilitado que cada año aumente el número de mujeres policías, sino que también poco a poco empieza a ser frecuente que ocupen puestos estratégicos o de mando, a pesar de que todavía no son consideradas una fuerza de iguales magnitudes que la de los varones, ni en jerarquía ni en número.

La incorporación de las mujeres a las fuerzas policiales se lleva a cabo de igual forma que la de los hombres. Consiste en un proceso de reclutamiento que se abre por medio de una convocatoria, donde se incorpora un número nutrido de aspirantes (según la necesidad de elementos y el proceso de reclutamiento) compuestos tanto de hombres como de mujeres, se realiza una serie de exámenes previos de perfil psicológico, médico y fisiológico, a partir de lo cual se estima si el aspirante cumple o no con los requisitos básicos para su evaluación (edad, peso, estatura, salud, estado mental, perfil socioeconómico, entre otros factores). Después, el(la) aspirante es programado(a) para la realización de sus evaluaciones de control de confianza, donde se aplica el famoso polígrafo, entre otras pruebas, que determinan si el aspirante es digno de confianza (desde una perspectiva muy genérica), para que la institución evaluadora -que puede ser del mismo estado o institución que desea reclutar- notifique los resultados. Si estos son aprobatorios entonces el(la) aspirante podrá ser incorporado(a) a la Academia para cursar un programa de formación inicial.

En esta etapa de formación tradicionalmente se ha pensado que las academias de formación policial confían en un ideal masculino en la policía, bajo el precepto histórico que representa que sea un "oficio de hombres" al cual se incorporarían años más tarde las mujeres. De manera que inicialmente las academias policiales se enfocaron en la preparación de ejercicios relacionados con fuerza física en su capacitación y dejaron de 
lado temas como la policía de proximidad, la resolución de problemas y las habilidades de comunicación interpersonal.

Actualmente, esta percepción se ha transformado, pues en las academias policiales se enfatiza en la importancia de la prevención más que en la reacción ante amenazas delincuenciales, lo que desplaza la idea de fuerza por la de administración de recursos como los de la comunicación y la previsión, aspectos en los cuales las mujeres policías muestran mejores habilidades. En muchos países latinoamericanos se ha comprobado que el uso de la fuerza no ha contribuido a disminuir el impacto de la incidencia delictiva; que las corporaciones policiales se han minado de la influencia y presencia narcotráfico; y que la militarización (como es el caso de la incorporación de la Guardia Nacional para realizar funciones de seguridad pública en México) tampoco ha disminuido la presencia ni la crueldad de los grupos delictivos.

De acuerdo con Arteaga (2000):

En general, la academia, a diferencia de los exámenes, sí representa un filtro para las mujeres que deciden ingresar a la policía. El ritmo de trabajo - particularmente el físico - representará un mecanismo de exclusión. Si se observan los porcentajes de deserción entre hombres y mujeres se ve cómo - en el caso que se analizó- casi el 50 \% de las mujeres desertaron, frente a un $3 \%$ de hombres, quienes desistieron principalmente por razones económicas ya que encontraron actividades mejor remuneradas. De 9 mujeres que se inscribieron, solo se graduaron 5. Las que desertaron argumentaron que era muy pesado el entrenamiento y que no lo aguantaban. De hecho, este fenómeno se repite en las policías de otros países. (pp. 79-80)

Otro estudio realizado con hombres y mujeres de la policía andaluza señala que 
uno de los datos donde mayor diferencia se observa entre mujeres y hombres es en aquel que hace referencia a la percepción de discriminación de género a la hora tanto de acceder como de promocionar en el Cuerpo. De esta forma, alrededor de la mitad (45.7\% en el acceso y $53.5 \%$ en la promoción) de las mujeres afirman que se han sentido discriminadas por el hecho de ser mujeres, mientras que un escaso porcentaje de hombres se han sentido de esta forma ( $7.4 \%$ en el acceso y $4.3 \%$ en la promoción), siendo este un resultado esperable porque la desigualdad estructural de género afecta principalmente a las mujeres dentro de un sistema patriarcal. (Gálvez et ál., 2015, pp. 13-14)

Las habilidades de fuerza o destreza física son solo una parte de las funciones que debe desarrollar la policía, lo verdaderamente importante radica en la posibilidad de mediar en conflictos interpersonales. Por todo ello resulta esencial que las instituciones de seguridad pública se esfuercen en cerrar la brecha de género que existe en los procesos de desarrollo policial, incentivando de una forma más amplia el reclutamiento femenino y garantizando condiciones igualitarias para su incorporación, a partir de mecanismos de medición y acceso ad hoc a las necesidades de la institución, sin dejar descuidar la evaluación de hombres y mujeres.

Las instituciones de seguridad pública deben entender y sensibilizarse ante la necesidad de generar una cultura institucional que garantice condiciones de respeto hacia los derechos humanos, especialmente para aquellos que protegen y garantizan los derechos de las mujeres y niñas y el acceso a una vida libre de todo tipo de violencia. En este sentido, se debe reconocer que la función policial es un eje transversal que se ocupa del cuidado y la preservación, pero al mismo tiempo de cumplir con los indicadores necesarios que sirvan de alarma para prever factores de riesgo para las mujeres, en un proceso que conciba la detección, identificación, 
intervención, atención, protección y prevención, para dar seguimiento específico a casos de violencia de género (Protocolo, 2020).

Por consiguiente, es vital que se reconozcan y preserven los derechos de las mujeres adentro y afuera de las instituciones, las mujeres policías deben contar con herramientas para empoderar su función frente a la ciudadanía y frente a sus compañeros, de manera que sea posible establecer condiciones equitativas de acceso a posiciones de poder y se fortalezcan aquellas áreas que permiten tener una vida laboral digna, con estricto respeto a sus derechos y garantías.

\section{Cultura institucional con perspectiva de género}

Aunque las mujeres configuran una fuerza menor dentro de las corporaciones policiales, representan una actividad verdaderamente relevante al interior de cualquier institución de seguridad pública. Contar con mujeres policías resulta esencial dentro de cualquier corporación policial, pues no solo se trata de una condición de género para incentivar los mecanismos de inclusión, igualdad laboral y transversalidad, sino que también confiere una serie de beneficios para la institución en su incorporación.

En las corporaciones policiales también resulta necesario formar una cultura institucional basada en la perspectiva de género y en la transversalidad de las condiciones laborales y de acceso igualitarias en la administración pública estatal, que permita identificar las áreas de oportunidad donde es preciso realizar un balance y un diagnóstico sobre las conductas o prácticas que incentiven el empoderamiento, liderazgo e inclusión de las mujeres en la toma de decisiones.

En el contexto de la administración pública estatal, las mujeres deben sortear toda clase de dificultades que complican su carrera de ascenso 
dentro de las estructuras de jerarquización laboral; pues, además de la carga de trabajo, en muchos casos las mujeres también desempeñan labores de madres, hijas, hermanas, cuidadoras, proveedoras, amas de casa o educadoras. Por lo tanto, es imprescindible que exista una cultura institucional que, en conjunto con los distintos órganos de gobierno, fomente estrategias y normativas que sirvan como catalizadoras de las impresiones, emociones y necesidades que surgen en los espacios laborales, con el propósito de frenar aquellas prácticas que generan la problemática del ascenso (Borbón, 2019).

De acuerdo con Pérez Gómez (1999), la cultura institucional de género se puede definir como un conjunto de significados y comportamientos que se produce al interior de las instituciones, que puede presentarse de forma arraigada, sutil o evidente, suele estar presente en la cotidianidad de las actividades y funciones que realizan los funcionarios públicos, y puede restringir o limitar las relaciones jerarquizadas de poder. Asimismo, se desarrollan de forma "natural" y se "normalizan" como parte de las creencias, prácticas, actividades, comportamientos e incluso dentro de los procesos, procedimientos y asignaciones de puestos o ascensos.

Lagarde (1996) señala que la perspectiva de género se estructura a partir de la ética y conduce a una filosofía poshumanista, pues critica la concepción androcéntrica de la sociedad que deja de lado a la mitad del género humano, es decir, a las mujeres. Por su parte, Lamas (1996) menciona que, en teoría, el género es comprendido como la simbolización o construcción cultural que hace referencia a la relación entre los sexos, es decir, que alude a las diferencias entre feminidad y masculinidad. La implementación de una cultura institucional con perspectiva de género posibilita visibilizar aquellas prácticas o conductas normalizadas al interior de las instituciones que no permiten generar condiciones 
igualitarias para sus trabajadores(as), y que se ven enraizadas con mayor fuerza dentro de las corporaciones policiales. Las prácticas fincadas en los estereotipos y roles de género inciden en las condiciones de desigualdad y en el éxito de las políticas públicas, así como en los bienes y servicios públicos que recibe la población (Patarroyo y Benavides, 2014).

Incorporar la perspectiva de género en las instituciones de seguridad pública hace parte esencial de las acciones afirmativas que se requieren para configurar condiciones igualitarias al interior de estas. Sin duda, el reto principal es que la incorporación de la perspectiva de género sea eficiente, pero sobre todo que involucre a todas y todos los miembros de la institución. De manera que paulatinamente este enfoque pueda permear todo el sistema en sus procesos no solo de selección, reclutamiento o incorporación, sino también en la toma de decisiones, en la asignación de puestos de mando y directivos, en el uso de lenguaje incluyente, en la sensibilización frente a los temas de género, en la creación de planes y programas con enfoque de género, en el objetivo de visibilizar todos los tipos de violencias que se pueden dar en el entorno laboral y fuera de este, y en la reflexión e interpretación sobre las condiciones personales, familiares o sociales que enfrentan las mujeres en el ejercicio de sus funciones.

Aun cuando existe un alto interés en incorporar la agenda de género dentro de las instituciones de administración pública, como las de seguridad, no basta con establecer protocolos, documentos o acuerdos, ni con la celebración de eventos, seminarios, cursos o talleres en la materia. Es preciso establecer una incorporación real de la perspectiva de género en la cultura institucional, que comienza de forma descendente y no ascendente, como se piensa comúnmente. En muchos de los casos, precisamente son los funcionarios que ocupan altos mandos quienes 
propician, replican o generan conductas y prácticas que alimentan una cultura machista, misógina y de violencia.

La construcción real de una cultura institucional que garantice condiciones igualitarias entre hombres y mujeres está relacionada con la visibilización de esas prácticas desiguales. Si dentro de la propia institución no se reconoce que existen serios problemas afianzados en la falta de políticas y procedimientos con enfoque de género, difícilmente la institución puede asumir prácticas sanas de convivencia laboral y de igualdad de condiciones entre hombres y mujeres. Popularmente se dice que lo que no se nombra no existe, lo que se aplica en el caso de la violencia de género, pues no se pueden cambiar prácticas que no han sido reconocidas, o bien aducir que son conductas que idealmente se desea romper pero que se practican en la cotidianidad.

En el caso de los(as) policías, el problema se agrava aún más debido a la función que cumplen en la prevención, erradicación y atención de la violencia de género, en muchas ocasiones, ellos(as) son el primer vínculo de la víctima con alguna institución de seguridad o que vela por la justicia, de manera que para romper con los ciclos de violencia es imprescindible sensibilizarlos para atender todos los tipos de abuso y agresión. La experiencia muestra que, al menos en México, las policías configuran una de las instituciones con menor porcentaje de confianza entre la ciudadanía (Inegi, 2019), no obstante, la corrupción, las prácticas ilegales y las prácticas de abuso y violencia por parte de los cuerpos de seguridad contra las mujeres propician la falta de confianza.

Asumir que la perspectiva de género no es un tema solo de mujeres, sino que hace parte de los retos que las instituciones de seguridad deben enfrentar para disminuir al mínimo las prácticas desiguales y permitir la incorporación de mujeres en funciones y actividades operativas y de 
mando, no únicamente en actividades administrativas o secretariales. Reducir al mínimo las condiciones de riesgo laboral asumidas por el acoso laboral y sexual, que terminan considerándose una condición para la asignación de ascensos y funciones, y desmitificar que la función policial es sinónimo únicamente de fuerza, reconocer las diferencias que naturalmente puedan tener ambos sexos y resaltar las fortalezas y aptitudes que cada uno de ellos puede aportar a la corporación son los grandes retos que una política de cultura institucional y perspectiva de género debe cumplir.

La discriminación de las mujeres por razones de género al interior de las instituciones de seguridad pública es una práctica usual que minoriza, estigmatiza, subordina, marginaliza y violenta sus derechos. Uno de los fundamentos principales de la incorporación de la perspectiva de género es garantizar que se asuma como una práctica habitual y cotidiana de las relaciones sociales. Para la sostenibilidad de este compromiso, la voluntad de los distintos actores involucrados debe traducirse en varios requisitos:

- Demostración de una disposición al cambio, que se traduce en voluntad y compromiso político.

- $\quad$ Formulación de políticas, leyes, normas y acciones específicas de intervención para hacer posible el logro de relaciones equitativas.

- Desarrollo de instrumentos técnicos y metodológicos capaces de incorporar esta nueva perspectiva en la planeación, seguimiento y evaluación.

- Innovar en las estructuras administrativas y de gestión.

- Sensibilizar y capacitar sobre el impacto diferenciado de la gestión gubernamental. 
- Disposición de recursos económicos suficientes para la planeación, ejecución, seguimiento y evaluación de las políticas institucionales internas y generales de equidad de género.

La implementación de estas medidas se ha realizado de forma gradual en las distintas instancias de la administración, en los tres niveles de gobierno y particularmente el problema se ha suscitado desde la adopción de programas por cuestiones de cumplimiento de estándares y medidas internacionales, que han recomendado al Estado mexicano cumplir con la incorporación de medidas de equidad y paridad, pero que en la práctica han tenido poco impacto, no solo al interior de las instituciones, sino en particular en relación con los(as) funcionarios(as).

Todavía existen condiciones de desigualdad que aumentan la brecha de género que existe en las corporaciones como la policía, en gran medida porque un factor relevante para el éxito de los planes y programas que buscan reducirla consiste en la sensibilización de los(as) funcionarios(as) públicos(as) frente a la violencia de género y los efectos que esta produce, así como sobre los daños estructurales que conlleva, no solo por la violencia física o feminicida - que es el nivel más extremo que puede existir-, sino respecto a la incorporación real de una cultura institucional con perspectiva de género, que permita establecer igualdad de condiciones laborales y salariales para hombres y mujeres.

La sensibilización de la agenda de género debe tener como punto focal un trabajo en conjunto para consolidar la igualdad sustantiva al interior de las instituciones de seguridad pública. Es preciso sumar esfuerzos no solo para capacitar y visibilizar los temas de violencia o perspectiva de género, sino que resulta fundamental incrementar los análisis al interior de las instituciones, evaluar las condiciones laborales de hombres y mujeres, los ascensos y grados asignados y cuántos de estos se han hecho de manera 
meritoria o por condiciones de género, reconocer la falta de espacios en posiciones de mando para mujeres e incorporar mecanismos transformadores - no necesariamente correctivos- en relación con las segregaciones de género.

Asimismo, es importante llevar la base teórica y de compromisos institucionales a una práctica real, es decir, cumplir a cabalidad las medidas y realizar seguimiento y evaluación, a través de instituciones u organismos de la sociedad civil, que funcionen como una especie de inspector o auditor para dichas transformaciones.

Particularmente, en el caso de las instituciones de seguridad pública, es preciso incorporar más mujeres a los cursos de formación inicial, lo que depende en gran medida del éxito de las convocatorias y proyectos de reclutamiento, pero que puede ser reforzado con campañas atractivas difundidas en los diversos medios de comunicación y entre la comunidad, para incrementar de forma considerable el número de mujeres participantes en los procesos de selección. También resulta pertinente adecuar los planes y programas educativos de formación inicial para atender las condiciones de cada sexo y potenciar las habilidades particulares de las mujeres policías, a partir de un enfoque basado en el liderazgo y empoderamiento de las posibles funcionarias, que no esté centrado exclusivamente en las funciones de fuerza y destreza - al no ser sus rasgos más notables, en comparación con sus homólogos varones-. La tradición histórica masculina que persigue a las corporaciones policiales necesita ser transformada, en aras de la incorporación equitativa de hombres y mujeres con vocación de servicio, y con una finalidad que va más allá del cumplimiento de cuotas de paridad o de género, sino como respuesta a la realidad de las instituciones policiales en países latinoamericanos, donde prevalecen bajos niveles de percepción de 
confianza en las instituciones, justificados en serios antecedentes de abuso, corrupción o ilegalidad. Precisamente, en este contexto las mujeres son quienes pueden ayudar en el saneamiento y mejoramiento de la confianza de la ciudadanía. No obstante, esto no implica que las mujeres sean una parte más sensible, vulnerable o amable de la policía, sino que su presencia en instituciones policiales en otros países del mundo ha demostrado ser altamente efectiva. Un estudio publicado por CNN reveló que

cuando las agencias de aplicación de la ley no reclutan, retienen ni promueven a las mujeres al mismo ritmo que los hombres, a pesar de que las investigaciones sugieren que, si lo hicieran, la nación vería muchas menos tragedias como las muertes de Floyd, Laquan McDonald o Eric Garner. (Fantz, 2020)

Además, el mismo estudio menciona que:

Las mujeres agentes son objeto de menos quejas ciudadanas y denuncias sostenidas de fuerza excesiva que sus colegas masculinos, según un estudio realizado en 2002. Esa investigación, considerada por expertos como una mirada histórica a las diferencias entre géneros en la policía, también muestra que las mujeres le cuestan menos dinero a los municipios y las agencias en pagos de responsabilidad civil en comparación con los hombres. El último hallazgo ha sido respaldado por investigaciones adicionales que muestran que las mujeres usan menos fuerza y son objeto de menos investigaciones locales y demandas. En Minneapolis, por ejemplo, solo 3 de más de 50 demandas resueltas por comportamiento entre 2010 y 2014 involucraron a mujeres acusadas de fuerza excesiva, según una investigación de la Radio Pública de Minnesota. (Fantz, 2020)

Asimismo, Harvey (2017) realizó un estudio con 400 miembros de la policía de Chattanooga, Tennessee, en Estados Unidos, donde demostró 
que las mujeres oficiales tienen más probabilidades de emplear ética del cuidado en sus métodos policiales, en comparación con sus colegas masculinos. El estudio, además, evaluó los procedimientos de la investigación existente en relación con múltiples aspectos del género y la policía. También exploró las relaciones de la policía con las víctimas y los delincuentes para identificar las diferencias en las maneras en que se relacionan las mujeres y los hombres agentes con los ciudadanos. Este análisis concluyó que, más allá de las diferencias de género, la incorporación de mujeres en la policía puede tener un amplio espectro de efectos positivos.

\section{Conclusiones}

La incorporación exitosa de nuevos integrantes precisa de forma previa y permanente el fomento de un ambiente laboral al interior de las instituciones policiales que garantice el desarrollo equitativo de las mujeres. En este sentido, se deben evitar las condiciones desiguales, el acoso sexual o laboral, la falta de empatía con las funciones personales o familiares y la falta de claridad en los parámetros de ascenso u obtención de logros, en un programa de carrera policial previamente reconocido e incorporado al interior de la institución.

Las instituciones de seguridad pública en América Latina aún enfrentan grandes retos en el esfuerzo de incorporar de forma correcta políticas y mecanismos de equidad y perspectiva de género. La cultura institucional debe atender el problema como parte de un mecanismo estructural que involucre todo el sistema jerárquico policial y el personal administrativo y auxiliar, con el propósito de generar un clima laboral adecuado y una política de estricto respeto a los derechos humanos y de cero tolerancia a las diversas formas de violencias que pueden visibilizarse dentro y fuera de la institución, en el entorno de lo público y lo privado. 
Durante años, las instituciones de seguridad pública han afianzado los estereotipos de género en función de las características que suelen tener hombres y mujeres, por eso es imprescindible crear una cultura institucional que, en conjunto con los distintos órganos de gobierno, pueda diseñar estrategias y normativas para la implementación del enfoque de género, que sirvan como catalizadores de las impresiones, emociones y necesidades que surgen en los espacios laborales, y que permitan eliminar aquellas prácticas que incentivan cualquier tipo de desigualdad o violencia basadas en género.

\section{Referencias}

Alarcón Sánchez, G. A., Díaz Ossa, S. Y. y Hernández Méndez, L. F. (2019). Las nociones de justicia que construyen las niñas en pleno siglo XXI. Via Inveniendi Et Iudicandi, 14(1), 67-89. https://doi.org/10.15332/s1909-0528.2019.0001.03

Ángel, A. (2019, 13 de septiembre). Solo 1 de cada 4 policías en México está capacitado para cuidar a la gente. México. Animal Político.

https://www.animalpolitico.com/2019/09/en-mexico-solo-1-de-cada-4-policiastiene-certificado-paratrabajar/\#: :text=Los\%20datos\%2oproporcionados\%20v\%C3\%ADa\%2otranspare ncia,Fiscal\%C3\%ADa\%20General\%20de\%20la\%20Rep\%C3\%BAblica)

Arteaga, N. (2000). El trabajo de las mujeres policías. El Cotidiano, 16(101), 74-83.

Blanco, C. (2020). El recordatorio a la determinación del contenido esencial de los derechos fundamentales atendiendo el escenario de la pandemia por el COVID 19, en el Estado colombiano. Novum Jus, 15(1), 17-40.

https://doi.org/10.14718/NovumJus.2021.15.1.2

Borbón Torres, J. A. (2019). La política de defensa y seguridad democrática en el Estado social de derecho. Revista IUSTA, 5O(1), 97-120. https://doi.org/10.15332/1900$\underline{0448.2019 .0050 .04}$

Revista IUSTA

ISSN: 1900-0448 | e-ISSN: 2500-5286 | DOI: https://doi.org/10.15332/25005286

N. ${ }^{0} 55$ | julio-diciembre del 2021 
Comisión Económica para América Latina y el Caribe [Cepal]. (2019). Solo en 2018 al menos 3529 mujeres fueron víctimas de feminicidio en 25 países de América Latina y el Caribe. https://www.cepal.org/es/comunicados/solo-2018-al-menos3529-mujeres-fueron-victimas-feminicidio-25-paises-america-latina

Comisión Nacional de Derechos Humanos [CNDH]. (2017). Estudio sobre la igualdad entre mujeres y hombres en materia de puestos y salarios en la Administración Pública Federal (APF).

https://www.cndh.org.mx/sites/default/files/doc/Informes/Especiales/Estudioigualdad-20180206.pdf

Connectas. (2020). Seis investigaciones para entender la violencia de género en América Latina. https://www.connectas.org/seis-investigaciones-para-entender-laviolencia-de-genero-en-america-latina/

Déziga Velázquez, P. (2019, 30 de octubre). Ser mujer policía. Animal Político. https://www.animalpolitico.com/el-blog-de-causa-en-comun/ser-mujer-policia/

Díaz, V. (2019, 21 de noviembre). El 2019 cerrará como el más violento. Milenio. https://www.milenio.com/policia/observatorio-nacional-ciudadano-2019-cerraraviolento\#: : text=El\%202019\%20cerrar\%C3\%A1\%20como\%20el\%20a\%C3\%B10\% 20m\%C3\%A1s\%20violento\%2C\%20debido\%20a,Observatorio\%2oNacional\%20Ci udadano\%20(ONC)

Faur, E. (2004). Masculinidades y desarrollo social. Las relaciones de género desde la perspectiva de los hombres. Unicef.

Feria Galbán, K. (2020). La seguridad y la salud en el trabajo. Una aproximación a través del derecho penal cubano. Revista IUSTA, (52), 15-50. https://doi.org/10.15332/25005286.5481

Fantz, A. (2020, 23 de junio). ¿Quieres reformar a la policía? Contrata más mujeres. CNN. https://cnnespanol.cnn.com/2020/06/23/quieres-reformar-a-la-policiacontrata-mas-mujeres/

Harvey, W. (2017). Gender differences in policing: a consideration of care ethics. [Tesis de grado, University of Tennessee]. Repositorio institucional de University of Tennessee.

https://scholar.utc.edu/cgi/viewcontent.cgi?article=1690\&context=theses

Revista IUSTA

ISSN: 1900-0448 | e-ISSN: 2500-5286 | DOI: https://doi.org/10.15332/25005286

N. ${ }^{0} 55$ | julio-diciembre del 2021 
Huertas, O. (2012). Violencia intrafamiliar contra las mujeres. Revista Logos Ciencia \& Tecnología, 4(1), 96-106.

idc Online. (2014, 5 de marzo). Mujeres más vulnerables al burnout.

https://idconline.mx/laboral/2014/03/05/mujeres-mas-vulnerables-al-burnout

Instituto Nacional de Estadística y Geografía [Inegi]. (2017). Encuesta Nacional sobre la Dinámica de las Relaciones en los Hogares (EDIREH).

https://www.inegi.org.mx/contenidos/programas/endireh/2016/doc/endireh2016 _presentacion_ejecutiva.pdf

Instituto Nacional de Estadística y Geografía [Inegi]. (2018) Primera Encuesta Nacional de Estándares y Capacitación Profesional Policial (ENCAP). https://www.inegi.org.mx/contenidos/saladeprensa/boletines/2018/EstSegPub/E NECAP2017.pdf

Instituto Nacional de Estadística y Geografía [Inegi]. (2019). Encuesta sobre el desempeño de las autoridades de seguridad pública y justicia. https://www.inegi.org.mx/temas/percepciondes/

Gálvez, L., Del Moral, L., Gallego, N., Matus, M. y Rodríguez P. (2016). La lenta normalización de la presencia de mujeres en cuerpos de seguridad. Análisis de los mecanismos y barreras de acceso y promoción de mujeres y hombres en el Cuerpo de las Policías Locales Andaluzas. Universidad Pablo de Olavide.

Gómez, A. y Herrera, D. (2018). La debida diligencia judicial y la protección de los derechos humanos de las mujeres en contextos de violencia. Iustitia, 16, 89-90.

Lagarde, M. (1996). Género y feminismo. Desarrollo humano y democracia. Horas y Horas.

Lamas, M. (1996). La perspectiva de género. En O. Mojarro Ávila y H. Carrizo Barrera (Cords.), Hablemos de sexualidad en la escuela secundaria: lecturas complementarias (pp. 217-229). Consejo Nacional de Población (Conapo).

Maldonado, G. (2017). La afiliación fraudulenta en el sistema de seguridad social integral colombiano. Via Inveniendi et Iudicandi, 12(2), 73-99. http://dx.doi. org/10.15332/s1909-0528.2017.0002.03 
Moreno, J., Meda, L., Rodríguez, M., Palomera, C. y Morales, M. (2006). El síndrome de burnout en una muestra de psicólogos mexicanos: prevalencia y factores sociodemográficos asociados. Psicología y Salud, 16(1), 5-13.

Patarroyo, S. y Benavides, P. (2014). Rupturas asignificantes: revisiones críticas en torno al derecho. Via Inveniendi et Iudicandi, 9(1), 7-31.

Pérez Gómez, A. (1999). La cultura escolar en la sociedad neoliberal. Morata.

Secretariado Ejecutivo del Sistema de Seguridad Pública [SESNSP]. (2020a). Incidencia delictiva del fuero común 2019. Instrumento para el registro, clasificación y reporte de delitos y las víctimas. $\mathrm{CNSP} / 38 / 15$. https://drive.google.com/file/d/1mDHUp5utLvucRrD6diuefgDtyqixIERX/view

Secretariado Ejecutivo del Sistema Nacional de Seguridad Pública [SESNSP]. (2020b). Modelo óptimo policial. http://sesnsp.com/mofp/

Protocolo Nacional de Actuación Policial para la atención a la violencia de género en contra las mujeres en el ámbito familiar. Secretaría de Seguridad y Protección Ciudadana. (2020). https://www.gob.mx/cms/uploads/attachment/file/614682/DOF__PROTOCOLO NACIONAL_DE ACTUACI_N_POLICIAL_PARA LA ATENCI N DE G NERO CONTRA LAS MUERES EN EL MBITO FAMILIAR VF.p $\underline{\mathrm{df}}$

Villasana, C. (2017, 11 de marzo). Mujeres policía, de la elegancia a la rudeza. El Universal. https://www.eluniversal.com.mx/entrada-deopinion/colaboracion/mochilazo-en-eltiempo/nacion/sociedad/2017/03/11/mujeres-policias 